\title{
Coronary Artery Dissection NHLBI Grade Clinical Classification
}

National Cancer Institute

\section{Source}

National Cancer Institute. Coronary Artery Dissection NHLBI Grade Clinical Classification. NCI Thesaurus. Code C119105.

A standardized grading system developed by the American College of Cardiology American Heart Association to describe coronary artery dissection. This grading system includes 6 classifications of dissection types A through F based on angiographic appearances. 